\title{
Mitigating Near-field Interference in Laptop Embedded Wireless Transceivers
}

\author{
Marcel Nassar, Kapil Gulati, Arvind K. Sujeeth, Navid Aghasadeghi, Brian L. Evans and Keith R. Tinsley
}

\begin{abstract}
In laptop and desktop computers, clocks and busses generate significant radio frequency interference (RFI) for the embedded wireless data transceivers. RFI is impulsive in nature. When detecting a signal in additive impulsive noise, Spaulding and Middleton showed a potential improvement in detection of $25 \mathrm{~dB}$ at a bit error rate of $10^{-5}$ when using a Bayesian detector instead of a standard correlation receiver. In this paper, we model impulsive noise using Middleton Class A and Symmetric Alpha Stable $(S \alpha S)$ models. The contributions of this paper are to evaluate (1) the performance vs. complexity of parameter estimation algorithms, (2) the closeness of fit of parameter estimation algorithms to measured RFI data from the computer platform, (3) the communication performance vs. computational complexity tradeoffs for the correlation receiver, Wiener filter, and Bayesian detector, and (4) the performance of myriad filtering in combating RFI interference modeled as $S \alpha S$ interference.
\end{abstract}

Index Terms-Alpha Stable, RFI Mitigation, Computational Platform, Impulsive Noise, Middleton Noise

\section{INTRODUCTION}

We address the reduction of RFI experienced by the wireless data communication transceivers deployed in a computation platform. Table I lists several wireless data communication standards and the computing platform subsystems (esp. clocks and busses) that have common spectral occupancy. The interference with wireless transceivers is not only due to the near-field coupling with radiation at frequencies of the driving clocks but also due to the harmonics produced by these subsystems.

RFI is a combination of independent radiation events, and predominantly has non-Gaussian statistics. Models used for RFI modeling include Middleton's Class A, B and C noise models (statistical-physical) [1] and Symmetric Alpha-Stable models (statistical) [2]. They are well-suited for modeling the predominantly non-Gaussian random processes that arise from the nonlinear phenomena that govern electromagnetic interference. Symmetric Alpha-Stable processes are included due to their mathematically tractable form for parameter estimators and communication detectors [3].

In this paper, we restrict our attention to combating Class A and $S \alpha S$ noise. We evaluate the detection performance of a correlation receiver, a Wiener filter, Bayes hypothesis testing [4], and a myriad filter [2].

Section II gives a brief introduction to Middleton noise models and Symmetric Alpha-Stable models. Section III describes different parameter estimation algorithms for

K. Gulati, M. Nassar, A. Sujeeth, N. Aghasadeghi, and B.L. Evans are with The University of Texas at Austin. K R. Tinsley is with the Intel Corporate Technology Group, Hillsboro, Oregon.

This research was supported by Intel.
TABLE I: Example of computer subsystems interfering with wireless standards [5], [6], [7]

\begin{tabular}{|l|l|l|}
\hline Standard & $\begin{array}{l}\text { Wireless } \\
\text { Networking }\end{array}$ & $\begin{array}{l}\text { Interfering Clocks } \\
\text { and Busses }\end{array}$ \\
\hline Bluetooth & $\begin{array}{l}\text { Personal Area Net- } \\
\text { work }\end{array}$ & $\begin{array}{l}\text { Gigabit Ethernet,PCI } \\
\text { Express Bus, LCD } \\
\text { clock harmonics }\end{array}$ \\
\hline $\begin{array}{l}\text { IEEE } 802.11 \mathrm{~b} / \mathrm{g} \\
\text { Fireless LAN (Wi- } \\
\text { Fi) }\end{array}$ & $\begin{array}{l}\text { Gigabit Ethernet, PCI } \\
\text { Express Bus, LCD } \\
\text { clock harmonics }\end{array}$ \\
\hline IEEE 802.16e & $\begin{array}{l}\text { High-Speed Wireless } \\
\text { LAN } \\
\text { Mi-Max) }\end{array}$ & $\begin{array}{l}\text { Gigabit Ethernet, PCI } \\
\text { Express Bus, LCD } \\
\text { clock harmonics }\end{array}$ \\
\hline IEEE 802.11a & $\begin{array}{l}\text { Wireless LAN (Wi- } \\
\text { Fi) }\end{array}$ & $\begin{array}{l}\text { PCI Express Bus, LCD } \\
\text { clock harmonics } \\
\text { PCI Express Bus, LCD } \\
\text { clock harmonics }\end{array}$ \\
\hline
\end{tabular}

these models, and their communication performance vs. computational complexity tradeoffs. Section IV quantifies the performance of the above methods applied to measured data provided by Intel. Last, Section V describes some RFI combating techniques for Class A and $S \alpha S$ noise, and provides a performance vs. complexity analysis of these algorithms. In this paper, "noise" and "interference" are used interchangeably as representing unwanted signals. We developed a MATLAB toolbox (www.ece.utexas.edu/ bevans/projects/rfi/software) that contains implementations of the algorithms mentioned in this paper and can be used to reproduce the obtained results.

\section{RFI NOISE MODELING}

Statistical-physical models provide accurate universal models for Electro-Magnetic Interference (EMI) from natural and human-made sources.

\section{A. Middleton Class A Model}

Middleton Class A noise model represents narrowband noise, i.e. when the interference spectrum is narrower than the receiver bandwidth. The Class A model is uniquely determined by two parameters [1]:

- $A_{A}$ (the overlap index), the product of the average number of emissions events impinging on the receiver per second and mean duration of a typical interfering source emission, e.g. $A_{A} \in\left[10^{-2}, 1\right]$ in [3].

- $\Gamma_{A}$ is the ratio $\left[\sigma_{G}^{2} / \Omega_{2 A}\right]$, where $\sigma_{G}^{2}$ is the intensity of the independent Gaussian component, $\Omega_{2 A}$ is intensity of the impulsive non-Gaussian component, e.g. $\Gamma_{A} \in$ $\left[10^{-6}, 1\right]$ in $[3]$. 
Envelope statistics for the Middleton class A model [1] are $\omega(\varepsilon)=2 e^{-A_{A}} \sum_{m=0}^{\infty} \frac{A_{A}^{m} \varepsilon e^{-\varepsilon^{2} / 2 \sigma_{m}^{2}}}{m ! \sigma_{m}^{2}}$ where $\sigma_{m}^{2}=\frac{\frac{m}{A_{A}}+\Gamma_{A}}{1+\Gamma_{A}}$.

\section{B. Symmetric Alpha Stable ( $S \alpha S)$ Model}

The practical applications of Class A and Class B models are limited due to the intractable form of their distributions [1]. Many authors have considered Symmetric Alpha Stable $(S \alpha S)$ models as an approximation to Middleton models [8], particulary in narrowband reception without a Gaussian component. In Section IV, we show that the Symmetric Alpha Stable $(S \alpha S)$ model serves as a good approximation for the physical phenomena underlying RFI. A random variable is said to have a $S \alpha S$ distribution if its characteristic function is of the form $\Phi(\omega)=e^{j \delta \omega-\gamma|\omega|^{\alpha}}$ where

- $\alpha$, the characteristic exponent, measures the "thickness" of the tail of the distribution, where $\alpha \in[0,2]$

- $\delta$, the localization parameter, is the mean when $1 \leq \alpha \leq$ 2 and the median when $0 \leq \alpha \leq 1$

- $\gamma$, the scale parameter or the dispersion, is similar to the variance of the Gaussian distribution, where $\gamma>0$

\section{PARAMETER Estimation}

In this section, we discuss various parameter estimation algorithms for both Class A and Symmetric Alpha Stable $(S \alpha S)$ noise models.

\section{A. Middleton Class A Model Parameter Estimation}

An efficient parameter estimation method for Class A model has been developed by Zabin and Poor based on the Expectation Maximization (EM) algorithm [3], [10]. They express the envelope probability density as a sum of weighted probability densities, under the constraint that the sum of the weights is equal to one. Let $\Theta=\left(A_{A}, K\right)$, where $K=A_{A} \Gamma_{A}$, denote the parameter set that has to be estimated.

The two steps, the expectation step (E-Step) and the maximization step (M-Step), of the expectation maximization algorithm given in [10] are hence given as follows:

- E-Step: Evaluate $Q\left(\Theta \mid \Theta^{(p)}\right)$, the expected value of the log-likelihood function

- M-Step: Determine $\Theta=\Theta^{(p+1)}$ to maximize $Q\left(\Theta \mid \Theta^{(p)}\right)$

Zabin and Poor give a closed form of the log-likelihood function $Q\left(\Theta \mid \Theta^{(p)}\right)$ [3]. The maximization step is then developed as a two-step iterative procedure [3], where we first maximize over $A_{A}$ assuming that $K$ is known and then vice-versa. The first minimization can be expressed as a polynomial of order 2 in $A_{A}$ and the second minimization can be expressed as a polynomial of order 4 in $K$ (after the linearizing approximation ). The two-step Maximization step can therefore be solved efficiently as it reduces to finding roots to polynomial equations of orders 2 and 4, respectively.

The performance of the EM Estimator developed by Zabin and Poor for Class A model has been shown in Fig. 1 for the estimation of parameter $A_{A}$. The number of iterations taken by the EM estimator to converge is proportional to $A$ and $K$. The number of iterations was observed to vary between 2 and 30 iterations to converge to a relative error

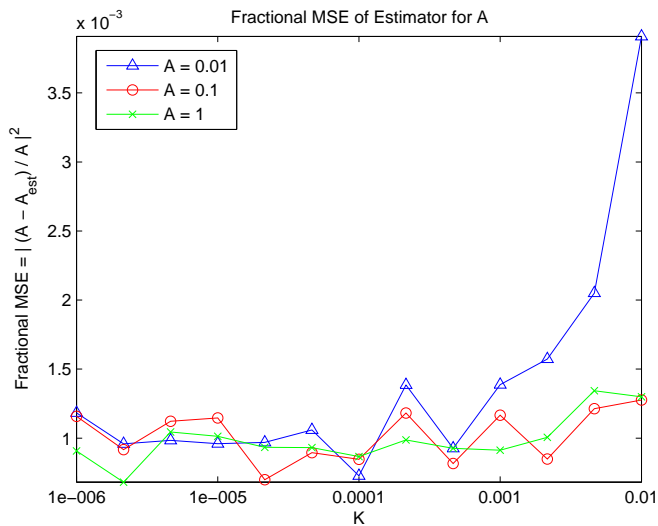

Fig. 1: Fractional MSE in estimates of parameter $A_{A}$ using EM algorithm [3] for Class A model

in successive estimates to be less than $10^{-7}$ in the range of interest. The estimates were calculated using $N=1000$ envelope data samples which were generated synthetically based on the envelope distribution. Note that the envelope probability density function (pdf) is expressed as an infinite sum and only the first 11 terms were used in simulations. The results were averaged over 50 Monte-Carlo simulation runs.

\section{B. Symmetric Alpha Stable (S $\alpha S$ ) Parameter Estimation}

An efficient, computationally fast estimator was developed by Tsihrintzis and Nikias [8]. It is based on the asymptotic behavior of extreme-order statistics, and is described next.

Let $X_{1}, X_{2}, \ldots, X_{N}$ be a collection of independent realizations of a random variable with the pdf $f$ and cumulative density function (cdf) $F$. Let $X_{M}$ and $X_{m}$ be the maximum and the minimum in the given sequence, respectively. Statistics of $X_{M}$ and $X_{m}$ are referred to as extreme-order statistics of the collection. For the alpha-stable model, it can be shown, using the theorem for Feasible Asymptotic Distribution for Extreme-Order Statistics, that the density of maxima and minima ( $f_{M}$ and $f_{m}$ ) approach the Frechet distributions as $N \rightarrow \infty$ [8]. Hence the estimators for the three parameters of the alpha-stable models are given by [8] as follows:

- Localization Parameter $(\delta)$ estimator is given by $\hat{\delta}=$ $\operatorname{median}\left(X_{1}, X_{2}, \ldots, X_{N}\right)$

- Characteristic Exponent $(\alpha)$ divides the data into $\mathrm{L}$ non-overlapping segments of equal length, computes the standard deviations of the natural logarithm of maximum $(=\bar{s})$ and minimum $(=\underline{s})$ of the data segments, and estimates $\hat{\alpha}=\frac{\pi}{2 \sqrt{6}}\left(\frac{1}{\bar{s}}+\frac{1}{\underline{s}}\right)$.

- Dispersion $(\gamma)$ estimator is given by $\hat{\gamma}=$ $\left[\frac{\frac{1}{N} \sum_{k=1}^{N}\left|X_{k}-\hat{\delta}\right|^{\frac{p}{\alpha}}}{C(p, \hat{\alpha})}\right]^{\frac{\alpha}{p}} \quad$ where $\quad C(p, \hat{\alpha})=\frac{1}{\cos \left(\frac{\pi}{2} p\right)} \frac{\Gamma\left(1-\frac{p}{\alpha}\right)}{\Gamma(1-p)}$ and $p$ is an arbitrary choice for the order $(0 \leq p \leq \hat{\alpha})$ of fractional moment.

The performance of the extreme-order statistics method [10] was observed when setting $\gamma=5$ (dispersion parameter) 


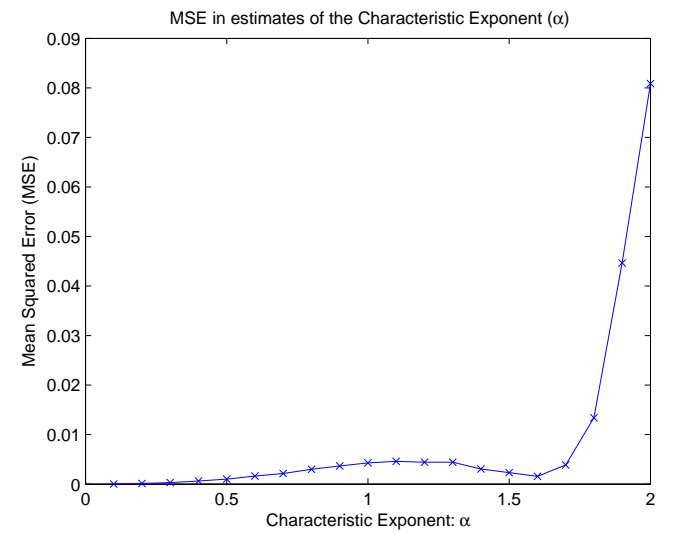

Fig. 2: MSE in the estimates of the Characteristic Exponent for $N=10000$ synthetic data samples, true parameters $\delta=$ 10 (localization), $\gamma=5$ (dispersion), $L=1250, p=\alpha / 3$.

and $\delta=10$ (localization parameter) and by varying $\alpha$ (characteristic exponent) over its entire range $(0 \leq \alpha \leq 2)$. Estimates were calculated using $N=10000$ data samples generated synthetically based on the characteristic function of symmetric alpha stable model. The data was segmented into $L=1250$ sets for the estimator for the characteristic exponent $\alpha$. Fractional lower-order moments of $1 / 3$ were used in the estimator for the dispersion parameter by choosing $p=\hat{\alpha} / 3$. The mean square error in the estimates for the characteristic exponent $(\alpha)$ is given in Fig. 2. The results were averaged over 100 Monte-Carlo simulation runs.

\section{Measured Data Fitting}

Measurements of radio frequency interference (RFI) on a computation platform were obtained from Intel Corporation. Measurement data was collected using a 20GSPS scope which represented actual radiated data. Four sets of measurement data were recorded in different configuration of the computation platform (i.e. different subsystems active etc). Each set of measurements contained 80000 samples of data. The noise was assumed to be "broadband", i.e. noise bandwidth was greater than the receiver bandwidth, and the radio was used to listen to the platform noise only (no data communication was being carried out). No further information was provided. We observed that the pdf of the measurements was symmetric. Hence the symmetric alpha stable model was chosen to model that measurement's pdf. The method of extreme order statistics yields a very good fit for the empirical data as can be seen from Fig. 3 .

\section{Filtering And Detection}

In this section, we evaluate the detection performance of the correlation receiver, the Wiener filter, the Bayes hypothesis testing, and the myriad filtering.

\section{A. Wiener Filtering}

The Wiener filter is the optimal linear filter in terms of minimizing the mean-squared error, and an finite impulse

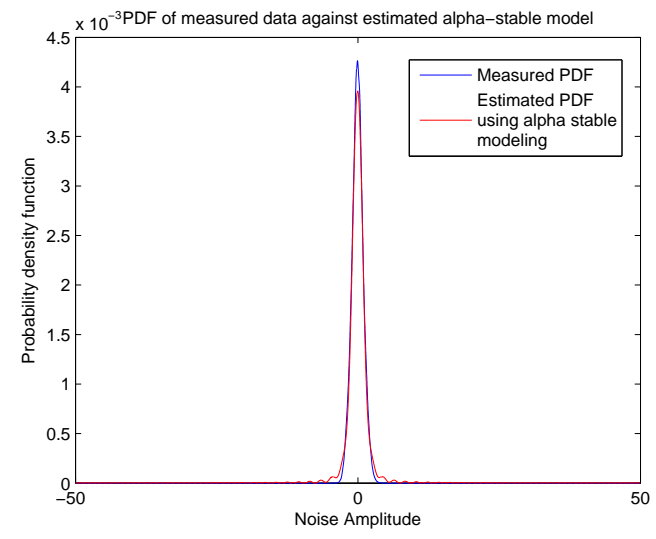

Fig. 3: PDF of measured data compared with the PDF of an estimated symmetric alpha stable model with $\alpha=1.5525$, $\gamma=0.5833$, and $\delta=-0.0393$.

response Wiener filter is designed using the famous WienerHopf equations. It assumes that the corrupting noise and desired signals are wide sense stationary.

\section{B. Coherent Bayes Detection}

Bayes detection is performed by choosing the hypothesis (bit) that maximizes the probability of receiving a signal given the sent hypothesis. Using Class A pdf, Spaulding and Middleton derived the optimal decision rule and its small signal approximation for coherent Class A detection [4].

The Bayes approach to detection is based on hypothesis testing. Spaulding and Middleton considered the case of digital binary signaling (two hypothesis $H_{1}$ and $H_{2}$ ) [4]. Using an additive noise model, the optimal Bayesian detection rule for a binary hypothesis case is given by: $\Lambda(\underline{X})=$ $\frac{p\left(H_{2}\right) p\left(\underline{X} \mid H_{2}\right)}{p\left(H_{1}\right) p\left(\underline{X} \mid H_{1}\right)} \geq_{H_{2}} 1$ where $\underline{X}$ is the received signal, and $p($. is obtained from Class A pdf.

By taking the Taylor series expansion of the Class A pdf and retaining the first-order terms of the gradient, Spaulding and Middleton were able to obtain a simplified expression for the detection rule, given below:

$$
x^{*}=\nabla_{X} \ln p_{Z}(\underline{X}) \cdot \triangle \underline{S}=\sum_{i=1}^{N}\left(s_{1 i}-s_{2 i}\right) \frac{d}{d x_{i}} \ln p_{Z}\left(x_{i}\right) \lessgtr_{H_{2}}^{H_{1}} 0
$$

This formula has a form similar to the standard correlation receiver for Gaussian noise with the exception of the nonlinearity $\frac{d}{d x_{i}} \ln p_{Z}\left(x_{i}\right)$ that precedes it.

\section{Myriad Filtering}

Myriad filters provide a filtering framework with high statistical efficiency in bell-shaped impulsive distributions like the $S \alpha S$ distribution. Gonzalez and Arce [2] have shown that myriad filters present important optimality properties along the $\alpha$-stable family. The myriad filter is a sliding window algorithm, that outputs the myriad of the sample window. The myriad of order $k$ of a set of samples $x_{1}, x_{2}, \ldots, x_{N}$ is defined as $\hat{\beta}_{k}=\arg \min _{\beta} \sum_{i=1}^{N} \log \left[k^{2}+\left(x_{i}-\beta\right)^{2}\right]$. The choice for $k$ can be determined by the following empirical formula 


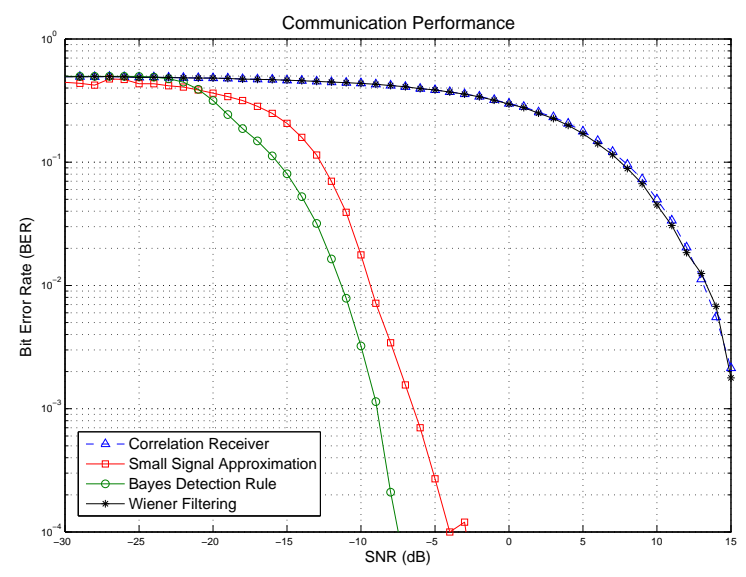

Fig. 4: Communication performance for Middleton Class A interference with $A_{A}=0.35$ and $\Gamma=0.5 \times 10^{-3}$.

$k(\alpha)=\sqrt{\frac{\alpha}{2-\alpha}} \gamma^{\frac{1}{\alpha}}$ where $\alpha$ and $\gamma$ are the parameters of the $S \alpha S$ noise [2].

\section{Performance Comparison}

Fig. 4 provides simulation results for a raised cosine pulse (10 samples per symbol period, 10 symbols period per symbol) sent through a memoryless channel (hence no intersymbol interference) and corrupted by Middleton Class A interference (with $A_{A}=0.35$ and $\Gamma=0.5 \times 10^{-3}$ ). The Wiener filter does not offer much improvement over the correlation receiver since it is suboptimal in non-Gaussian noise. The improvement in communication performance by using the Bayes detection rule is approximately $25 \mathrm{~dB}$ for a bit error rate of $10^{-3}$, and $20 \mathrm{~dB}$ for the small signal approximation. On the other hand, Fig. 5 compares the communication performance in the presence of additive $\mathrm{S} \alpha \mathrm{S}$ noise (with parameters $\alpha=0.5, \gamma=1$ and $\delta=0$ ). Since the second order moment of alpha stable processes do not exist, we compare the bit error rate (BER) against the generalized SNR, defined as $G S N R=10 \log \frac{E s}{\gamma}$, where Es denotes the signal power and $\gamma$ is the dispersion parameter of $\mathrm{S} \alpha \mathrm{S}$ process. The myriad filtering is preceded by the parameter estimation for $\mathrm{S} \alpha \mathrm{S}$ and followed by the correlation receiver. A gain of $5 d B$ is observed at a BER of $10^{-1}$.

\section{E. Complexity vs. Performance Comparison}

The complexity of the Bayesian detection is $\Theta(N M K U)$ operations to perform detection, due to the complex pdf function for Class A noise, where $N$ is the number of samples per symbol, $M$ is the number of terms we retain from the infinite series, $U$ is the cardinality of the signaling space and $K$ is the number of operations required to compute a transcendental function (an exponential in this case). The Wiener filer on the other hand requires $\Theta(N T)$ operations to compute its output, where $T$ is the number of taps of the Wiener filter. The correlation receiver in the given setting only requires $\Theta(N)$ operations. Taking these complexities and the results of Section V-D, it is noticed that additional

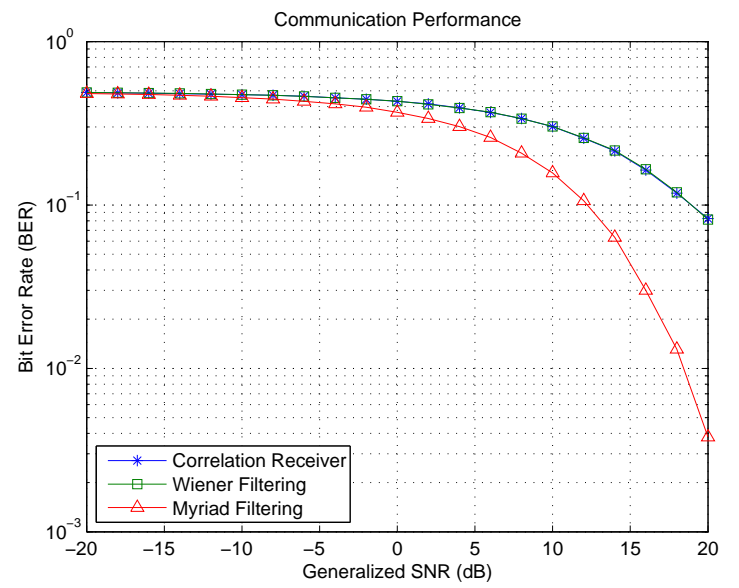

Fig. 5: Communication performance for $S \alpha S$ additive noise with $\alpha=1.5, \gamma=1$, and $\delta=0$.

TABLE II: Complexity vs. performance comparison.

\begin{tabular}{|c|c|c|}
\hline Method & Computational Complexity & Detection Performance \\
\hline Bayesian & $\Theta(N M K U)$ & High \\
\hline Correlation & $\Theta(N)$ & Low \\
\hline Wiener & $\Theta(N T)$ & Low \\
\hline
\end{tabular}

gain comes at the cost of higher complexity. The results are summarized in Table II.

\section{REFERENCES}

[1] D. Middleton, "Non-gaussian noise models in signal processing for telecommunications: New methods and results for class a and class b noise models," IEEE Trans. on Info. Theory, vol. 45, no. 4, pp. 1129-1149, 1999.

[2] J. G. Gonzalez and G. R. Arce, "Optimality of the myriad filter in practical impulsive-noise enviroments," IEEE Trans. on Signal Processing, vol. 49, no. 2, pp. 438-441, February 2001.

[3] S. M. Zabin and H. V. Poor, "Efficient estimation of class a noise parameters via the em [expectation-maximization] algorithms," IEEE Trans. on Info. Theory, vol. 37, no. 1, pp. 60-72, January 1991.

[4] A. Spaulding and D. Middleton, "Optimum reception in an impulsive interference enviroment-part i: Coherent detection," IEEE Trans. on Comm., vol. 25, no. 9, pp. 910-923, 1977.

[5] P. Havarasan, M. Haines, H. Skinner, and F. Justice, "Current and future emi challenges at intel and how to manage them," Proc. IEEE Int. Symposium on Electromagnetic Compatibility, vol. 1, pp. 281-283, August 2000.

[6] E. A. Morse, "A method for emi evaluation of notebook computer liquid crystal display panels while eliminating the contribution of computer generated emi," Proc. IEEE Int. Symposium on Electromagnetic Compatibility, pp. 343-346, August 1995.

[7] T. M. Zeeff, T. H. Hubing, J. L. Drewniak, R. E. Dussroff, and T. P. V. Doren, "Emc analysis of an 18" lcd monitor," Proc. IEEE Int. Symposium on Electromagnetic Compatibility, vol. 1, pp. 169173, August 2000.

[8] G. A. Tsihrintzis and C. L. Nikias, "Fast estimation of the parameters of alpha-stable impulsive interference," IEEE Trans. on Signal Processing, vol. 44, no. 6, pp. 1492-1503, June 1996.

[9] D. Middleton, "Procedures for determining the properties of the first-order canonical models of class a and class b electromagnetic interference," IEEE Trans. on Electromagnetic Compatibility, vol. 22, pp. 190-208, August 1979.

[10] J. Bilmes, "A gentle tutorial of the em [expectation-maximization] algorithm and its application to parameter estimation for gaussian mixture and hidden markov models," Manuscript, Int. Computer Science Institute, 1998. 